\title{
Complete Pathologic Response After Neoadjuvant Chemoradiotherapy for Esophageal Cancer Is Associated With Enhanced Survival
}

\author{
James M. Donahue, MD, Francis C. Nichols, MD, Zhuo Li, MS, David A. Schomas, MD, Mark \\ S. Allen, MD, Stephen D. Cassivi, MD, Aminah Jatoi, MD, Robert C. Miller, MD, Dennis A. \\ Wigle, MD, PhD, K. Robert Shen, MD, and Claude Deschamps, MD \\ Divisions of General Thoracic Surgery, Biostatistics, Radiation Oncology, and Medical Oncology, \\ Mayo Clinic, Rochester, Minnesota
}

\begin{abstract}
Background-Neoadjuvant chemoradiotherapy followed by esophagogastrectomy has become the standard of care for patients with locally advanced esophageal cancer. This report analyzes our experience with this treatment approach.

Methods-From January 1998 through December 2003, all patients from a single institution receiving neoadjuvant chemoradiotherapy followed by esophagogastrectomy were reviewed for operative mortality, morbidity, long-term survival, and factors affecting survival. Only patients preoperatively staged with both computed tomographic scans and endoscopic ultrasound were included.
\end{abstract}

\begin{abstract}
Results-There were 162 patients (142 men, 20 women), and the median age was 61 years (range, 22 to 81 years). Histopathology was adenocarcinoma in 143 patients and squamous cell in 19 . Pretreatment clinical stage was II in 28 patients (17\%), III in 111 (68\%), and IV (M1a) in 23 (14\%). Ivor Lewis esophagogastrectomy was the most common procedure, occurring in 132 patients. Operative mortality and morbidity was $4.9 \%$ and $37 \%$, respectively. Pathologic response was complete in 42 patients (26\%), near complete in 27 (17\%), partial in 88 (54\%), and unresectable in $5(3 \%)$. Five-year survival for overall, complete, near complete, and partial response patients was $34 \%, 55 \%, 27 \%$, and $27 \%$, respectively $(p=0.013)$. Patients whose lymph nodes were rendered free of cancer showed improved overall and disease-free survival compared with patients having persistently positive lymph nodes $(p=0.019)$.
\end{abstract}

Conclusion-Esophagogastrectomy after neoadjuvant chemoradiotherapy can be performed with low mortality and morbidity. Patients with complete pathologic response have significantly improved long-term survival compared with patients with near complete and partial responses. Future efforts should be directed at understanding determinants of complete responses.

The incidence of carcinoma of the esophagus is increasing and is the sixth leading US cause of male cancer death [1]. Most patients present with locally advanced disease. Historically, surgery alone resulted in 5-year survival rates of $10 \%$ to $20 \%$ [2-4]. Neoadjuvant chemoradiotherapy (nCRT) followed by surgical resection (nCRTSR) has become the standard of care for locally advanced esophageal cancer. This report reviews our single-institution experience with nCRTSR. 


\section{Material and Methods}

From our prospectively maintained database, we retrospectively reviewed all patients who underwent nCRTSR for esophageal cancer between January 1998 and December 2003 at Mayo Clinic, Rochester, Minnesota. Only patients whose pretreatment staging included both computed tomography and endoscopic ultrasonography (EUS) were eligible for study. Positron emission tomography (PET) became routine at our institution in 2001. Medical records were reviewed for age, sex, symptoms, histopathology, nCRT regimen, comorbidities, operation, histopathologic response, mortality, morbidity, length of hospitalization, adjuvant therapy, and long-term survival. Mayo Foundation's Institutional Review Board approved this study.

Neoadjuvant chemoradiotherapy consisted of two cycles of chemotherapy and concomitant radiotherapy. Radiotherapy included 28 daily fractions ( $180 \mathrm{cGy}$ ), 5 days/week for 5,040 cGy total dose. Chemotherapy consisted of 5-fluorouracil as a continuous 96 -hour infusion (1,000 $\mathrm{mg} / \mathrm{m}^{2}$ per day) and cisplatin $\left(75 \mathrm{mg} / \mathrm{m}^{2}\right.$ per day) as a daily bolus for 1 hour. Chemotherapy was given on the first and last 4 days of radiotherapy. A 4- to 6-week recovery period followed nCRT. Patients were considered to have completed nCRT if they finished two chemotherapy cycles and radiotherapy. Dose reductions during the second chemotherapy round were not considered criteria to rule nCRT incomplete. We have previously described our techniques for esophagogastrectomy [2,5-7]. Staging was by the American Joint Committee for Cancer Staging TNM classification [8].

Operative mortality included all deaths within 30 days of operation and those who died later but during the same hospitalization. The Social Security Death Index was used for survival data unavailable in medical records. All deaths were considered esophageal cancer related unless another cause was identified. After esophagogastrectomy, patients were classified as (1) complete pathologic response (CP) —-microscopic absence of any viable tumor, (2) near complete pathologic response (NCP) - microscopic foci of viable tumor cells in an otherwise necrotic specimen with no tumor remaining in resected lymph nodes, and (3) partial pathologic response (PP)—macroscopic residual viable tumor at primary site and/or positive lymph nodes.

Descriptive statistics for categorical variables are reported as frequency and percentage, and continuous variables are reported as mean (standard deviation) or median (range) as appropriate. Long-term survival was estimated using the Kaplan-Meier survival method, and 5-year estimates and 95\% confidence intervals (95\% CI) are reported [9]. The starting point in survival estimation was hospital discharge date, and date of death or last follow-up was the end point. The association of individual variables with survival was assessed using the logrank test for categorical variables and the Cox proportional hazards model for continuous variables and for the multivariate model $[10,11]$. The multivariable model considered univariately significant variables $(p<0.05)$ with model selection using the stepwise method (backward and forward methods resulted in the same model). Disease-free survival was analyzed with similar statistical methods with unresectable patients excluded. All statistical tests were two-sided with a probability value of 0.05 for statistical significance. To report our entire experience with patients having nCRTSR, we included some previously reported patients $[12,13]$. Importantly, this report has much stricter pre-nCRT clinical staging criteria than our earlier reports, and some earlier reported patients were excluded from this study. This study recognizes distinct post-nCRTSR pathologic categories besides identifying factors affecting long-term survival.

\section{Results}

One hundred ninety-four patients were treated by nCRTSR. Thirty patients were excluded because they did not undergo EUS, and 2 patients were excluded for refusal to participate in 
research. Thus, our final cohort included 162 patients (142 men, 20 women). Median age was 61 years (range, 22 to 81 years). Comorbidities included diabetes in 14 patients, coronary artery disease in 13, and both in 4 . Twenty-seven patients (16.7\%) had a greater than 20 pack-year smoking history. Barrett's esophagus was present in 57 patients (35.2\%).

All patients had pretreatment computed tomography and EUS. Positron emission tomography became available in 2001 and was obtained in 57 patients. Histopathologic designation was adenocarcinoma in 143 patients and squamous cell in 19. Tumor location was lower esophagus in 139 patients and mid-esophagus in 23. Endoscopic ultrasonography showed suspicious lymph nodes in 150 patients (92.5\%); EUS-directed fine-needle aspiration (EUS-FNA) of those lymph nodes was performed in 116 patients (71.6\%). Endoscopic ultrasonography FNA was not performed if FNA necessitated primary tumor transgression. Lymph node EUS-FNA was positive for malignancy in 92 patients (79.3\%). Endoscopic ultrasonography revealed pathologically suspicious celiac axis lymphadenopathy (M1a disease) in 23 patients, and EUSFNA was performed in 19 of these patients. Biopsied celiac lymph nodes demonstrated malignancy in 17 patients $(89.5 \%)$. Table 1 shows the pretreatment clinical stage of the patients.

Our standard chemotherapy regimen was used in 156 patients, and 5-fluorouracil, carboplatin, and paclitaxel was used in 6 patients. Chemotherapy was completed in 145 patients (89.5\%); 23 had a dose reduction during the second round. Median radiotherapy dose was 5,040 cGy (range, 1,260 to 5,400 cGy). A feeding tube was required during nCRT in 48 patients, and included an operative jejunostomy tube in 21 , percutaneous endoscopic gastrostomy in 16 , percutaneous endoscopic jejunostomy in 7 , and a nasojejunal tube in 4 . There were no feeding tube-related complications. Hospitalization for treatment toxicities occurred in 65 patients (40.1\%). Thrombotic complications including deep venous thrombosis occurred in 21 patients, and pulmonary embolism occurred in 7 . Deep venous thrombosis was in the upper extremity in 20 patients. Four patients with pulmonary embolism had vena cava filters placed before esophagogastrectomy. Median time interval from completion of nCRT to esophagogastrectomy was 44 days (range, 21 to 107 days).

Five patients (3.1\%) had unresectable cancer as a result of metastatic disease. Metastases were liver, omentum, pancreas, porta hepatis, and splenic artery lymph node in 1 patient each. Type of esophagogastrectomy was at the surgeon's discretion and included Ivor Lewis in 132 patients, extended esophagectomy (McKeown) in 22, transhiatal in 2, and left

thoracoabdominal approach in 1 . Anastomosis was hand-sewn in 155 patients and stapled in 7. Postoperatively, 71 patients had a feeding jejunostomy tube. Median number of lymph nodes resected was 15 (range, 3 to 50). Thirteen patients received intraoperative radiation therapy.

Three patients (1.9\%) had positive surgical margins. Table 2 shows pathologic stage data; 115 patients (70.9\%) were downstaged. Complete pathologic response occurred in 42 patients (25.9\%), NCP in $27(16.7 \%)$, and PP in 88 (54.3\%). Of 150 N1 lymph node-positive patients before nCRT, 79 (52.7\%) were downstaged to N0 postoperatively. Of 23 M1a lymph nodepositive patients, 17 (73.9\%) were downstaged. For these M1a patients, 15 patients (65.3\%) had PP, $6(26.1 \%)$ had CP, and 1 (4.3\%) each had NCP or unresectable cancer. Of the 15 PP M1a patients, 5 had persistent M1a disease. Table 3 shows pretreatment stage and response to nCRT. Figure 1 shows overall 5-year survival estimates based on pre-therapy stage.

There were 8 operative deaths (4.9\%). Cause of death was pneumonia in 4 patients, sepsis after anastomotic leak in 3, and bleeding gastric ulcer in 1. Complications occurred in 60 patients (37.0\%): atrial fibrillation in 26, pneumonia in 23, anastomotic leak in 19, wound infection in 13 , chylothorax in 8 , vocal cord paralysis in 7 , and myocardial infarction in 1. Regarding anastomotic leaks, 13 (68.4\%) were only radiographic findings and nonoperatively managed, whereas 6 required reoperation. Five patients had successful leak repair, and 1 had esophageal 
exclusion. Five patients $(62.5 \%)$ with chylothorax required reoperation. Seventy-one patients required blood transfusion within 48 hours of surgery. Median number of units transfused was 2 (range, 1 to 6 units). Median hospitalization was 11 days (range, 3 to 143 days). Adjuvant chemotherapy was only given to patients with unresectable cancer or those subsequently exhibiting recurrent cancer.

Follow-up was complete for all patients and ranged from 49 days to 7.5 years (median, 2.0 years). Overall median survival for operative survivors was 2.1 years. Median survival for 5 unresectable patients was 217 days (range, 58 to 675 days). At last follow-up, 58 patients were alive at a median of 4.1 years (range, 73 days to 7.4 years). Overall 5 -year survival was $33 \%$ ( $95 \%$ CI, $26.1 \%$ to $42.6 \%$ ). Overall 5-year survival for pathologically downstaged patients was $38.1 \%$ (95\% CI, $29.4 \%$ to $49.2 \%$ ), compared with $20.6 \%$ (95\% CI, $10.3 \%$ to $41.4 \%$ ) for patients not downstaged $(p=0.017)$. Figure 2 shows overall survival for patients on the basis of whether $\mathrm{CP}$ was achieved. Overall 5-year survival for $\mathrm{CP}$ was $55 \%$ (95\% CI, $41.5 \%$ to $74.0 \%$ ) compared with $26.1 \%$ (95\% CI, $18.6 \%$ to $36.6 \%$; $p=0.013$ ) when any residual cancer (NCP or PP) remained. We compared survival between NCP and PP patients. Overall 5-year survivals for patients with NCP and PP were 27.4\% (95\% CI, $13.9 \%$ to 54.1\%) and $27.3 \%$ (95\% CI, $18.6 \%$ to $40.1 \%)$, respectively ( $p=0.98$; Fig 3 ).

Figure 4 shows the effect of pathologic lymph node status on overall survival. Overall 5-year survival in patients rendered $\mathrm{N} 0$ was $43 \%$ (95\% CI, 32.7\% to 56.4\%) compared with $24 \%$ when $\mathrm{N} 1$ disease persisted (95\% CI, $15.4 \%$ to $38.8 \% ; p=0.019)$. When CP patients were excluded, overall 5-year survival for the remaining N0 patients was $32.2 \%$ (95\% CI, 19.9\% to 52.2\%), and for the remaining N1 patients it was $24.5 \%$ (95\% CI, $15.4 \%$ to $38.8 \% ; p=0.20$ ).

Univariate analysis was performed to determine factors associated with improved survival. Factors analyzed included age, sex, smoking history, pretreatment stage, histopathology, presence of Barrett's esophagus, completion of nCRT, transfusion requirement, achievement of $\mathrm{CP}$, and postresection lymph node status. Univariate analysis revealed age younger than 60 years $(p=0.03)$, female sex $(p=0.03)$, postresection N0 status $(p=0.02)$, and achievement of $\mathrm{CP}(p=0.01)$ were all associated with improved survival. In a multivariate model, age younger than 60 years $(p=0.02)$ and achievement of $\mathrm{CP}(p=0.005)$ were associated with improved survival.

Given the profound impact achieving CP had on survival, univariate analysis of the same above factors was performed to determine which patients were likely to achieve CP. In that analysis, female sex $(p=0.04)$ and squamous histopathology $(p=0.0003)$ were predictive of CP. The small numbers of female patients and those with squamous histopathology precluded further meaningful analyses. Overall 5-year survival was 31.3\% (95\% CI, 23.8\% to 41.0\%) for patients with adenocarcinoma and $51.4 \%$ (95\% CI, 30.8\% to $95.8 \%$ ) for patients with squamous histopathology $(p=0.103)$.

Information regarding tumor recurrence was available for 123 patients $(82.5 \%)$. Recurrences occurred in 63 patients (51.2\%). Median time to recurrence was 276 days (range, 46 days to 4.7 years). Recurrences were distant in 53 patients, and locoregional in 10.

Overall disease-free median survival was 1.7 years, and 5-year disease-free survival for all patients was 32\% (95\% CI, 24.4\% to 41.1\%). Five-year disease-free survival for CP patients was $49 \%$ (95\% CI, $34.9 \%$ to $68.2 \%$ ) compared with $25.4 \%$ (95\% CI, $17.6 \%$ to $36.6 \%$ ) when any residual cancer remained ( $p=0.035$; Fig 5 ). Similar to overall survival, there was no difference in disease-free survivals for NCP and PP patients. Five-year disease-free survival was $28 \%$ ( $95 \%$ CI, $14.2 \%$ to $54.9 \%$ ) for NCP patients and $24.2 \%$ (95\% CI, $15.3 \%$ to $38.2 \%$ ) for PP patients $(p=0.91)$. Figure 6 shows 5-year disease-free survival on the basis of posttreatment pathologic lymph node status. Five-year disease free-survival was $41.3 \%$ (95\% 
CI, $31.6 \%$ to $54.1 \%$ ) for patients pathologically N0 and $19.7 \%$ (95\% CI, $10.9 \%$ to $35.6 \%$ ) for patients pathologically $\mathrm{N} 1(p=0.015)$. When CP patients were excluded, 5-year disease-free survival for the remaining N0 patients was $34.9 \%$ (95\% CI, $22.9 \%$ to $53.2 \%$ ), and for the remaining $\mathrm{N} 1$ patients it was $19.7 \%(95 \% \mathrm{CI}, 10.9 \%$ to $35.6 \% ; p=0.14)$.

\section{Comment}

Prognosis for patients with locally advanced esophageal cancer is grim. Neoadjuvant chemoradiotherapy followed by surgical resection has become common treatment for locally advanced esophageal cancer. Although 156 of our patients received the standard nCRT regimen, 6 patients received 5-fluorouracil, carboplatin, paclitaxel, and radiation preoperatively as part of the North Central Cancer Treatment Group N0044 trial [14]. Despite the N0044 trial's 35\% CP, this protocol demonstrated significant treatment-related toxicities. Five (83.3\%) of these patients required hospitalization compared with 60 (38\%) having standard nCRT. We have discontinued use of the N0044 trial regimen.

Lacking a clinical trial, our approach for managing esophageal cancer has been based on clinical stage. Currently, all potentially resectable patients have computed tomography, PET, and EUS. If evidence of distant metastatic disease exists, confirmatory biopsies are performed. Endoscopic ultrasonography FNA is routinely performed on all suspicious lymph nodes provided the primary tumor is not transgressed. Patients thought to have T1NOM0 or T2NOM0 esophageal cancer are offered esophagogastrectomy. Patients with T1N1M0, T2N1M0, T3N1M0, T3N0M0, T4N0M0, and T4N1M0 disease are offered nCRTSR, and patients with M1a disease are considered for nCRTSR. In this study, pretreatment stage was not a significant predictor of improved survival, likely because of the small number of lymph node- negative patients. Restaging after nCRT includes computed tomography and PET. Positron emission tomography was not available for the entire study, and thus restaging did not routinely use PET. None of the ultimately unresectable patients had PET.

Besides nCRT, intraoperative radiation therapy was used in 13 patients. These patients had bulky tumors with 7 staged as either T4 or M1a by EUS. The decision for intraoperative radiation therapy was made preoperatively by both the surgeon and radiation oncologist. None of these 13 patients had CP. Eleven of these cases were performed before 2002, and intraoperative radiation therapy is no longer used at the same time as nCRTSR. Intraoperative radiation therapy may be beneficial as salvage therapy in the rare patient with a long diseasefree interval and isolated local recurrence [15].

Our early nCRTSR experience has been previously reported $[12,13]$. This current series contains a larger patient cohort with median 2-year follow-up compared with our earlier 11month median follow-up. Besides the more accurate pretreatment clinical staging, the current review stratifies nCRTSR patients on degrees of pathologic response and pathologic lymph node status, not just whether CP or PP was achieved. This study demon-strated a statistically significant overall and disease-free survival advantage for CP patients. Finally, this review addresses morbidity experienced by patients during nCRT and our approach to preoperative nutrition.

A CP ranging from 15\% to 30\% is reported in most nCRTSR series [12,16-29]. Our 25.9\% $\mathrm{CP}$ is similar. Our study's 5-year 55\% overall and $49 \%$ disease free-survival is an improvement over the historical $10 \%$ to $20 \%$ 5-year surgery-alone survival rates; however, the survival benefit for nCRT patients not achieving CP is difficult to elucidate. After nCRTSR, the importance of accurately stratifying patients is critical in determining prognosis. The American Joint Committee for Cancer Staging TNM classification system does not include a category for CP, making its usefulness in post-nCRTSR staging problematic [29,30]. The simplest way 
to categorize patients after nCRTSR is whether CP is achieved, but this rationale fails to capture patient subsets with residual disease that might have improved survival. Rohatgi and colleagues [31] have correlated treatment failure patterns with the degree of nCRT response. However, they did not consider the resected lymph node status, which traditionally is a key part of pathologic staging [32,33]. Others have analyzed both lymph node status and degree of pathologic response [27,29]. In these reports, similar to ours, regardless of the nCRT response, pathologic lymph node-negative (N0) patients had improved survival compared with patients with residual positive lymph nodes. When patients with CP were removed from the N0 subset, survival was still superior to the N1 subset, $32 \%$ versus $24 \%$, although the difference was no longer significant $(p=0.2)$.

A significant difference in survival existed between our patients with CP versus $\mathrm{PP}(p=0.01)$; however, the difference between CP and NCP was not significant $(p=0.08)$. Moreover, for patients with any residual cancer (NCP or PP), we were unable to detect any survival differences. This discrepancy is likely related to our small number of patients with NCP compared with the threefold larger PP group. Perhaps owing to limitations in power, we were unable to find subsets of PP patients who enjoyed a significant survival advantage. However, survival trends indicated that patients with minimal residual disease and negative lymph nodes did better than only PP with residual N1 disease.

Forty percent of our patients required hospitalization for toxicities during nCRT; however, most patients $(89.5 \%)$ were able to complete nCRT and undergo esophagogastrectomy. Besides nCRT-related morbidity, concerns have been raised regarding operative risk after nCRT. Our series' operative mortality of $4.9 \%$ is comparable to that reported by others [25,34], but higher than our previously reported $1.4 \%$ to $2.3 \%$ esophagogastrectomy mortality rates that contained few nCRT patients $[2,6]$. Our 37\% morbidity compares favorably with our previous reports of $37.7 \%$ to $47 \%$ morbidity without nCRT [2,6]. Similarly, Lin and colleagues [35] showed no increased morbidity with nCRT compared with surgery alone. Our current series' 3-year survival of $41 \%$ for patients with residual tumor is nearly identical to the $40 \%$ to $42 \% 3$-year survival reported in other recent series for surgery alone $[13,25]$. The current broad usage of nCRT seems justified based on the markedly improved survival of patients who achieve CP and the similar survival and morbidity rates for patients with residual tumor after nCRT compared with patients not having nCRT before surgery.

Efforts are being directed at preoperatively identifying variables predictive of CP. Agarwal and colleagues [36] suggested that patients with Barrett's esophagus or symptomatic gastroesophageal reflux disease have lower rates of CP and overall survival. We detected no differences in either survival or CP in our patients with Barrett's esophagus. Our study showed female sex or squamous histopathology was associated with increased likelihood of CP. Rohatgi and associates [37] identified higher CP in women. Kesler and colleagues [25] found a higher rate of CP with squamous cell cancer; however, Rohatgi and colleagues [38] did not. The utility of PET in predicting CP after nCRT is currently being investigated by us and others. Except for ruling out distant metastatic disease, the results of restaging PET are too early to draw conclusions. Future insight regarding the biologic predictors of achieving $\mathrm{CP}$ will require advancements in genomic and proteonomic analysis.

In conclusion, this nonrandomized, retrospective review demonstrates that nCRTSR can be performed with low mortality and morbidity. Patients with a CP have significantly improved long-term survival compared with patients having only an NCP or PP. Patients successfully downstaged from N1 to N0 lymph node status enjoy improved survival. Given the lack of data from a modern, randomized trial, these data add to the accumulated experience regarding both the efficacy and limitations of treating locally advanced esophageal cancer patients with 
nCRTSR. Future efforts need to be directed at understanding the determinants of achieving a CP after nCRT.

\section{Abbreviations}

CI

CP

EUS

EUS-FNA

$\mathrm{NCP}$

nCRT

nCRTSR

PET

PP confidence intervals

complete pathologic response

endoscopic ultrasound

endoscopic ultrasound fine-needle aspiration

near complete pathologic response

neoadjuvant chemoradiotherapy

neoadjuvant chemoradiotherapy followed by surgical resection

positron emission tomography

partial pathologic response

\section{References}

1. Cancer Facts \& Figures 2007. American Cancer Society, Inc; 2007.

2. Visbal AL, Allen MS, Miller DL, Deschamps C, Trastek VF, Pairolero PC. Ivor Lewis esophagogastrectomy for esophageal cancer. Ann Thorac Surg 2001;71:1803-1808. [PubMed: 11426751]

3. Jaroszewski, DE.; Deschamps, C.; Gunderson, LL.; Lanza, LA.; Trastek, VF.; Pairolero, PC. Cancer of the esophagus. In: Kelly, KA.; Sarr, MG.; Hinder, RA., editors. Mayo Clinic gastrointestinal surgery. Philadelphia, PA: WB Saunders; 2004. p. 57-73.

4. Chak, B.; Forastiere, AA. Neoadjuvant therapy for esophageal cancer. In: Yang, CY.; Cameron, DE., editors. Current therapy in thoracic and cardiovascular surgery. Philadelphia, PA: Mosby; 2004. p. 356-360.

5. Behzadi A, Nichols FC, Cassivi SD, Deschamps C, Allen MS, Pairolero PC. Esophagogastrectomy: the influence of stapled versus hand-sewn anastomosis on outcome. J Gastrointest Surg 2005;9:10311040. [PubMed: 16269373]

6. Vigneswaran WT, Trastek VF, Pairolero PC, Deschamps C, Daly RC, Allen MS. Extended esophagectomy in the management of carcinoma of the upper thoracic esophagus. J Thorac Cardiovas Surg 1994;107:901-906.

7. Vigneswaran WT, Trastek VF, Pairolero PC, Deschamps C, Daly RC, Allen MS. Transhiatal esophagectomy for carcinoma of the esophagus. Ann Thorac Surg 1993;56:838-844. [PubMed: 8215660]

8. American Joint Committee on Cancer: AJCC cancer staging handbook. New York: Springer-Verlag; 2002.

9. Kaplan E, Meier P. Nonparametric estimation from incomplete observations. J Am Stat Assoc 1958;53:457-481.

10. Cox DR. Regression models and life tables (with discussion). J Royal Stat Soc Series B 1972;34:187220.

11. Peto R, Peto J. Asymptotically efficient rank invariant procedures (with discussion). J Royal Stat Soc Series A 1972;135:185-207.

12. Donington JS, Miller DL, Allen MS, Deschamps C, Nichols FC, Pairolero PC. Tumor response to induction chemoradiation: influence on survival after esophagectomy. Eur J Cardiothorac Surg 2003;24:631-637. [PubMed: 14500086]

13. Donington JS, Miller DL, Allen MS, Deschamps C, Nichols FC, Pairolero PC. Preoperative chemoradiation therapy does not improve early survival after esophagectomy for patients with 
clinical stage III adenocarcinoma of the esophagus. Ann Thorac Surg 2004;77:1193-1199. [PubMed: 15063233]

14. Jatoi A, Martenson JA, Foster NR, et al. Paclitaxel, carboplatin, 5-fluorouracil, and radiation for locally advanced esophageal cancer. Am J Clin Oncol 2007;30:507-513. [PubMed: 17921712]

15. Miller RC, Haddock MG, Gunderson LL, et al. Intraoperative radiotherapy for treatment of locally advanced and recurrent esophageal and gastric adenocarcinomas. Dis Esophagus 2006;19:487-495. [PubMed: 17069594]

16. Walsh TN, Noonan N, Hollywood D, Kelly A, Keeling NP, Hennessy TP. A comparison of multimodal therapy and surgery for esophageal adenocarcinoma. N Engl J Med 1996;335:462-467. [PubMed: 8672151]

17. Urba SG, Orringer MB, Turrisi A, Iannettoni M, Forastiere A, Strawderman M. Randomized trial of preoperative chemoradiation versus surgery alone in patients with locoregional esophageal carcinoma. J Clin Oncol 2001;19:305-313. [PubMed: 11208820]

18. Le Prise E, Etienne PL, Meunier B, et al. A randomized study of chemotherapy, radiation therapy, and surgery versus surgery for localized squamous cell carcinoma of the esophagus. Cancer 1994;73:1779-1784. [PubMed: 8137201]

19. Franklin R, Steiger Z, Vaishampayan G, et al. Combined modality therapy for esophageal squamous cell carcinoma. Cancer 1983;51:1062-1071. [PubMed: 6401596]

20. Leichman L, Steiger Z, Seydel HG, et al. Preoperative chemotherapy and radiation therapy for patients with cancer of the esophagus: a potentially curative approach. J Clin Oncol 1984;2:75-79. [PubMed: 6538224]

21. Seydel HG, Leichman L, Byhardt R, et al. Preoperative radiation and chemotherapy for localized squamous cell carcinoma of the esophagus: a RTOG study. Int J Radiat Oncol Biol Phys 1988;14:3335. [PubMed: 3335460]

22. Poplin E, Fleming T, Leichman L, et al. Combined therapies for squamous-cell carcinoma of the esophagus, a Southwest Oncology Study Group (SWOG-8037). J Clin Oncol 1987;5:622-628. [PubMed: 3559653]

23. Forastiere AA, Orringer MB, Oerez-Tamayo C, Urba SG, Zahurak M. Preoperative chemoradiation followed by transhiatal esophagectomy for carcinoma of the esophagus: final report. J Clin Oncol 1993;11:1118-1123. [PubMed: 8501498]

24. Adelstein DJ, Rice TW, Becker M, et al. Use of concurrent chemotherapy, accelerated fractionation radiation, and surgery for patients with esophageal carcinoma. Cancer 1997;80:1011-1020.

[PubMed: 9305700]

25. Kesler KA, Helft PR, Werner EA, et al. A retrospective analysis of locally advanced esophageal cancer patients treated with neoadjuvant chemoradiation therapy followed by surgery or surgery alone. Ann Thorac Surg 2005;79:1116-1121. [PubMed: 15797035]

26. Rohatgi P, Swisher SG, Correa AM, et al. Characterization of pathologic complete response after preoperative chemoradiotherapy in carcinoma of the esophagus and outcome after pathologic complete response. Cancer 2005;104:2365-2372. [PubMed: 16245310]

27. Schneider PM, Baldus SE, Metzger R, et al. Histomorphologic tumor regression and lymph node metastases determine prognosis following neoadjuvant radiotherapy for esophageal cancer Implications for response classification. Ann Surg 2005;242:684-692. [PubMed: 16244542]

28. Hammoud ZT, Kesler KA, Ferguson MK, et al. Survival outcomes of resected patients who demonstrate a pathologic complete response after neoadjuvant chemoradiation therapy for locally advanced esophageal cancer. Dis Esophagus 2006;19:69-72. [PubMed: 16643172]

29. Rizk NP, Venkatraman E, Bains MS, et al. American Joint Committee on Cancer staging system does not accurately predict survival in patients receiving multimodality therapy for esophageal adenocarcinoma. J Clin Oncol 2007;25:507-512. [PubMed: 17290058]

30. Swisher SG, Hofstetter W, Wu TT, et al. Proposed revision of the esophageal cancer staging system to accommodate pathologic response (pP) following preoperative chemoradiation (CRT). Ann Surg 2005;241:810-817. [PubMed: 15849517]

31. Rohatgi PR, Swisher SG, Correa AM, et al. Failure patterns correlate with the proportion of residual carcinoma after preoperative chemoradiotherapy for carcinoma of the esophagus. Cancer 2005;104:1349-1355. [PubMed: 16130133] 
32. Rizk NP, Venkatraman E, Park B, et al. The prognostic importance of the number of involved lymph nodes in esophageal cancer; implications for revisions of the American Joint Committee on Cancer Staging System. J Thorac Cardiovasc Surg 2006;132:1374-1381. [PubMed: 17140960]

33. Hofstetter W, Correa AM, Bekele N, et al. Proposed modification of nodal status in AJCC esophageal cancer staging system. Ann Thorac Surg 2007;84:365-373. [PubMed: 17643602]

34. Berger AC, Farma J, Scott WJ, et al. Complete response to neoadjuvant chemoradiotherapy in esophageal carcinoma is associated with significantly improved survival. J Clin Oncol 2005;23:4330-4337. [PubMed: 15781882]

35. Lin FC, Durkin AE, Ferguson MK. Induction therapy does not increase surgical morbidity after esophagectomy for cancer. Ann Thorac Surg 2004;78:1783-1789. [PubMed: 15511475]

36. Agarwal B, Swisher SG, Ajani J, et al. Differential response to preoperative chemoradiation and surgery in esophageal adenocarcinomas based on presence of Barrett's esophagus and symptomatic gastroesophageal reflux. Ann Thorac Surg 2005;79:1716-1723. [PubMed: 15854962]

37. Rohatgi PR, Correa AM, Swisher SG, et al. Gender-based analysis of esophageal cancer patients undergoing preoperative chemoradiation: differences in presentation and therapy outcome. Dis Esophagus 2006;19:152-157. [PubMed: 16722991]

38. Rohatgi PR, Swisher SG, Correa AM, et al. Histologic subtypes as determinants of outcome in esophageal carcinoma patients with pathologic complete response after preoperative chemoradiotherapy. Cancer 2006;106:552-558. [PubMed: 16353210]

\section{DISCUSSION}

DR WAYNE L. HOFSTETTER (Houston, TX): Dr Donahue, congratulations on an excellent presentation, and I also congratulate the authors on the excellent surgical outcomes presented in this paper. This manuscript represents another opportunity for us to examine the additional benefit of neoadjuvant therapy in patients who are undergoing surgical resection for locally advanced esophageal cancer. As we are lacking in significant phase III data, this paper serves as another bit of armor indicating that patients who are downstaged after neoadjuvant therapy, especially those patients who are rendered PCR (pathologically complete resection) or nodenegative, perform better than expected compared to historical controls for similarly staged patients treated with surgery.

A couple of comments and questions. Your results are very consistent with those that have been previously published in regards to the benefit of complete pathologic response on survival. What your data addresses but incompletely answers is whether or not downstaging which is incomplete will result in some survival benefit. You have chosen to group your patients into three categories: PCR, NCR, PPR, and have shown that there is an impressive survival benefit to those who have had a PCR but those that have had a near complete response (NCR) or a partial response (PPR) fall onto a lower curve with no statistical difference between the two lesser groups. You have suggested, therefore, that the incomplete responders lack a significant survival benefit. Our group has shown that there is potentially another subgroup of patients who may respond somewhat but are essentially nonresponders, resulting in a significant volume of viable tumor at the time of resection. Our pathologists measure the extent of histoviability of the remaining tumor with proven reproducibility, and we have found that those patients that have greater than $50 \%$ tumor viability fall onto a significantly lower survival curve compared to patients who have microscopic residual (NCRs) or are partial responders with less than 50\% viable tumor. This suggests that although not as robust as the PCR group, a partial responder may enjoy an intermediate survival benefit, therefore potentially gaining benefit from being downstaged at the primary tumor. So I would like you to comment on that.

Another question is, and we are all guilty of this, do you have a total denominator in terms of an intent to treat? Is it possible for you to go into your database and retrieve on an intent to treat basis all of those patients who underwent neoadjuvant therapy and therefore include in your analyses those that potentially did not make it to surgery? 
Again, I congratulate you on a great presentation.

DR DONAHUE: Thank you very much, Dr Hofstetter. In regard to the first question, we were disappointed to find that patients who had a near complete response did not enjoy an improved survival benefit over patients who had only a partial response. However, there were only 27 patients in this study who met criteria that we defined for a near complete response. So in part, the statistically insignificant difference in survival between the near complete and partial response groups may have something to do with the small numbers. In addition, our pathologists did not quantify the amount of remaining viable tumor, making our classification somewhat subjective. This may have led to some inaccuracy in classifying responses as near complete or partial.

As to the second question regarding the denominator, we tried quite hard with the medical oncologists to try to come up with the actual denominator but were unable to do so. This was due in part to the fact that many of the patients at the Mayo Clinic have their therapy at other institutions and then come back for surgery, and I think we lost a fair number of them to followup.

DR JOSEPH B. ZWISCHENBERGER (Lexington, KY): All of us in the room are faced with making decisions on management in a multidisciplinary setting, and we all have patients with multiple comorbidities. I think your $5 \%$ operative mortality and your $37 \%$ morbidity rate are believable. The real question is, what is the actual role of surgery? When you show a complete response, you show an improved survival. If you are looking at a patient with what appears to be a complete response and trying to decide whether to subject them to that $5 \%$ mortality and that $37 \%$ morbidity, would they have done just as well without the surgery?

DR DONAHUE: That is an excellent question, Dr Zwischenberger, and I think something we are going to have to address specifically in the future. I think there is a parallel with patients with colorectal cancer who are treated with neoadjuvant therapy and have similar complete response rates. The complete response in those patients is able to be determined very well prior to surgery, and there is a rather good published experience with not operating on those patients. I think in esophageal cancer we still cannot assess whether or not a patient has had a complete response very well without surgery. In addition, we know from data on chemoradiation alone that those patients have fairly high local recurrence rates. So, I think at this point we still have to operate on all patients who do not progress on neoadjuvant therapy.

DR MARK J. KRASNA (Towson, MD): Congratulations, Jim, excellent presentation. The data from the CALGB 9781 actually will be coming out in print later this year in JCO (Journal of Clinical Oncology). Although it is a small number, that is a prospective randomized trial which was powered to detect a very small difference, in fact down to a $30 \%$ difference, in survival. So hopefully Dr Zwischenberger will have some additional data.

Just a quick question for you. We currently have now been using chemoradiation before surgery for all of our N1 patients, so TX, N1, and all of our T3 patients regardless of node stage, and I am curious now in Mayo, given this information, if you have a specific algorithm, how do you handle that in-between group? If you have a clinical esophageal ultrasound patient that is a T2, so they are not quite T3, and there are no obvious lymph nodes or you have tried EUSFNA (endoscopic ultrasonography fine-needle aspiration) and the lymph nodes are negative, is that a patient who you subject to neoadjuvant chemoradiation or does that patient get surgery alone?

DR DONAHUE: Thanks, Dr Krasna. Those patients are, as you say, kind of on the borderline, but the current practice at the Mayo Clinic for a T2N0 clinically staged patient is to proceed directly with surgery. 


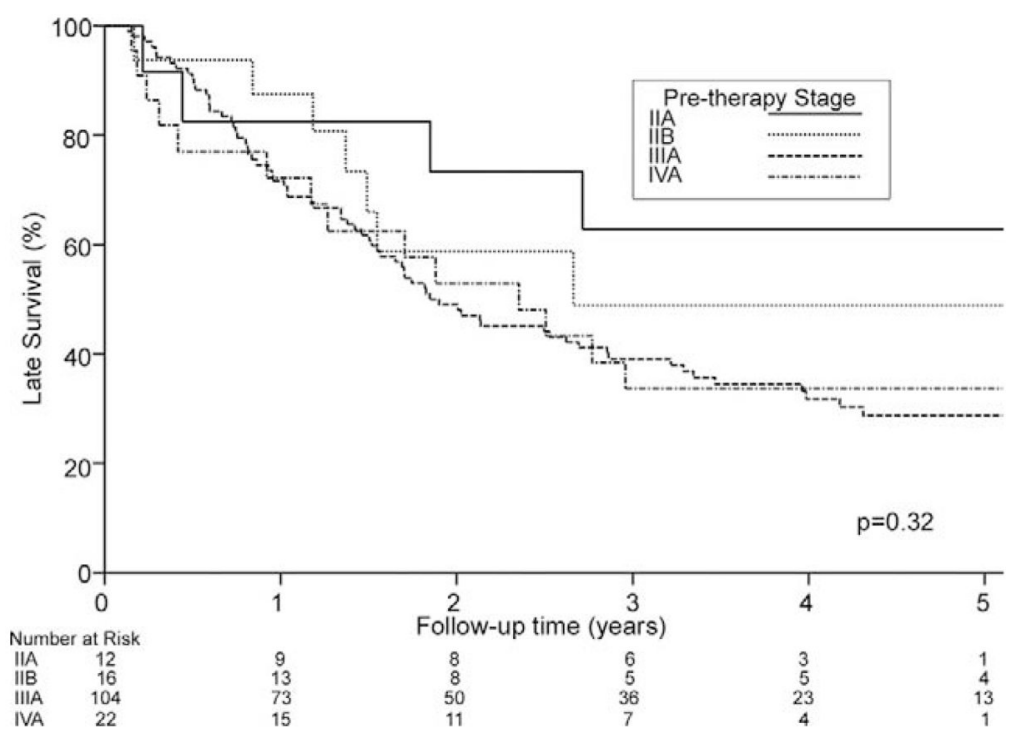

Fig 1.

Probability of overall survival (death from any cause) in 154 operative survivors based on pretherapy stage. Zero time represents hospital discharge date. IIA versus IIB, $p=0.44$; IIA versus III, $\mathrm{p}=0.10$; IIA versus IVA, $\mathrm{p}=0.142$. 


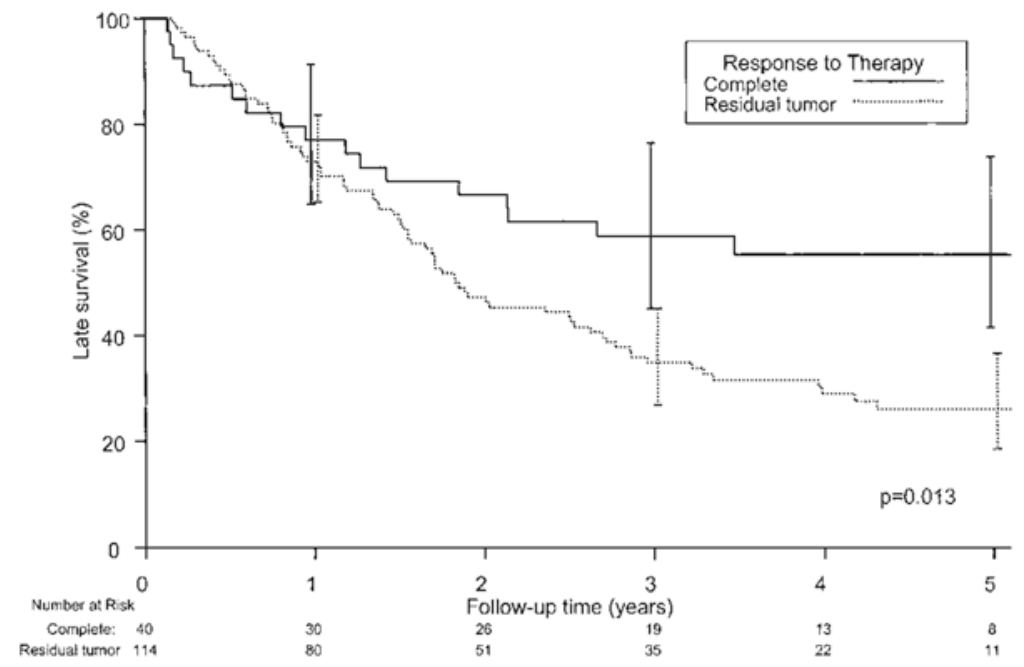

Fig 2.

Probability of overall survival (death from any cause) based on response to therapy after neoadjuvant chemoradiotherapy followed by surgical resection. Forty patients had complete pathologic response, 114 patients had some degree of residual tumor (near complete, partial pathologic response, or unresectable cancer). Zero time represents the hospital discharge date. 


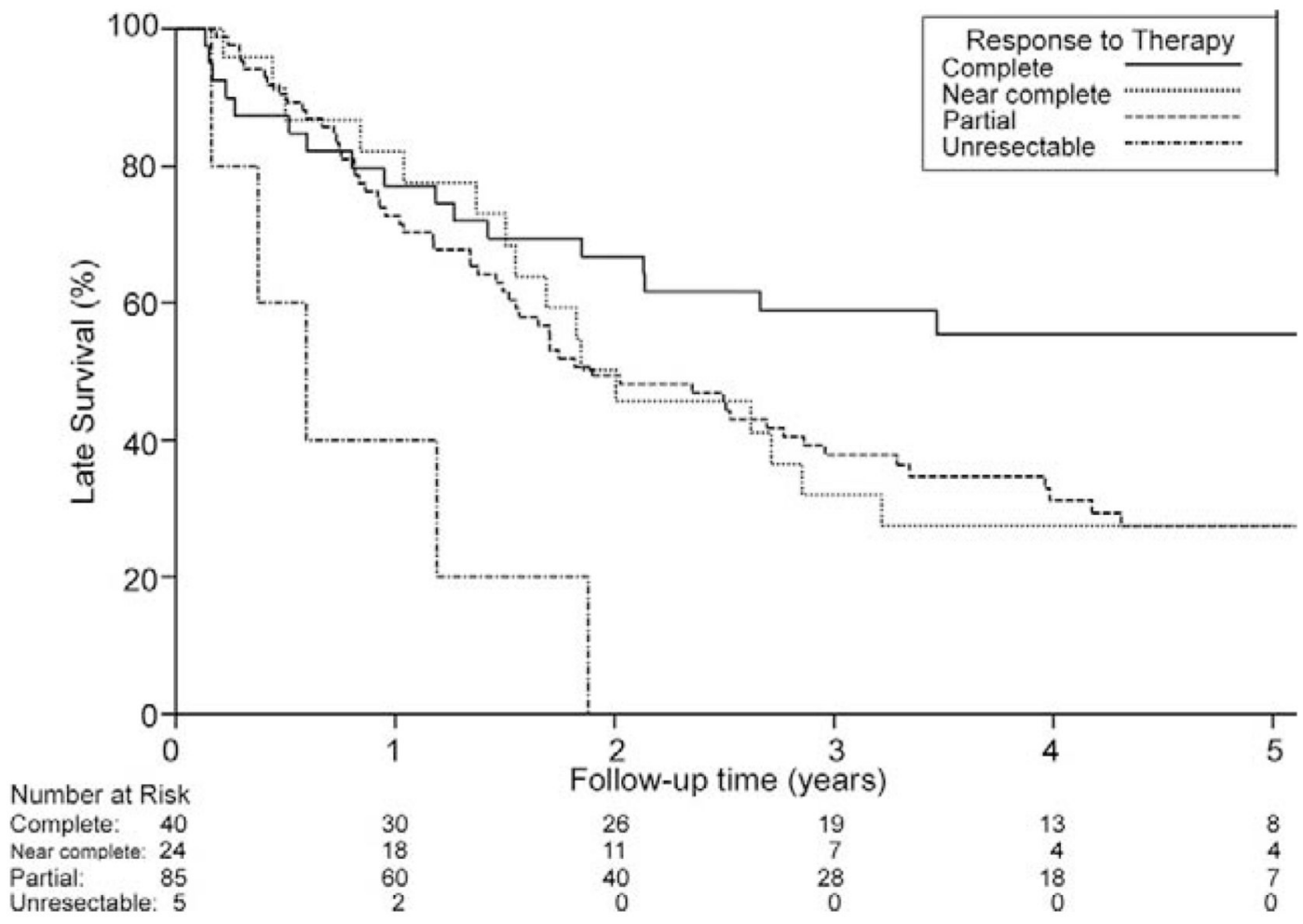

Fig 3.

Probability of overall survival (death from any cause) based on response to therapy after neoadjuvant chemoradiotherapy followed by surgical resection. Zero time represents hospital discharge date. Complete versus near complete, $\mathrm{p}=0.08$; complete versus partial, $\mathrm{p}=0.026$; complete versus unresectable, $\mathrm{p}=0.0002$, near complete versus partial, $\mathrm{p}=0.978$. (Complete $=$ complete pathologic response; Near complete $=$ near complete pathologic response; Partial $=$ partial pathologic response; Unresectable $=$ unresectable cancer.) 


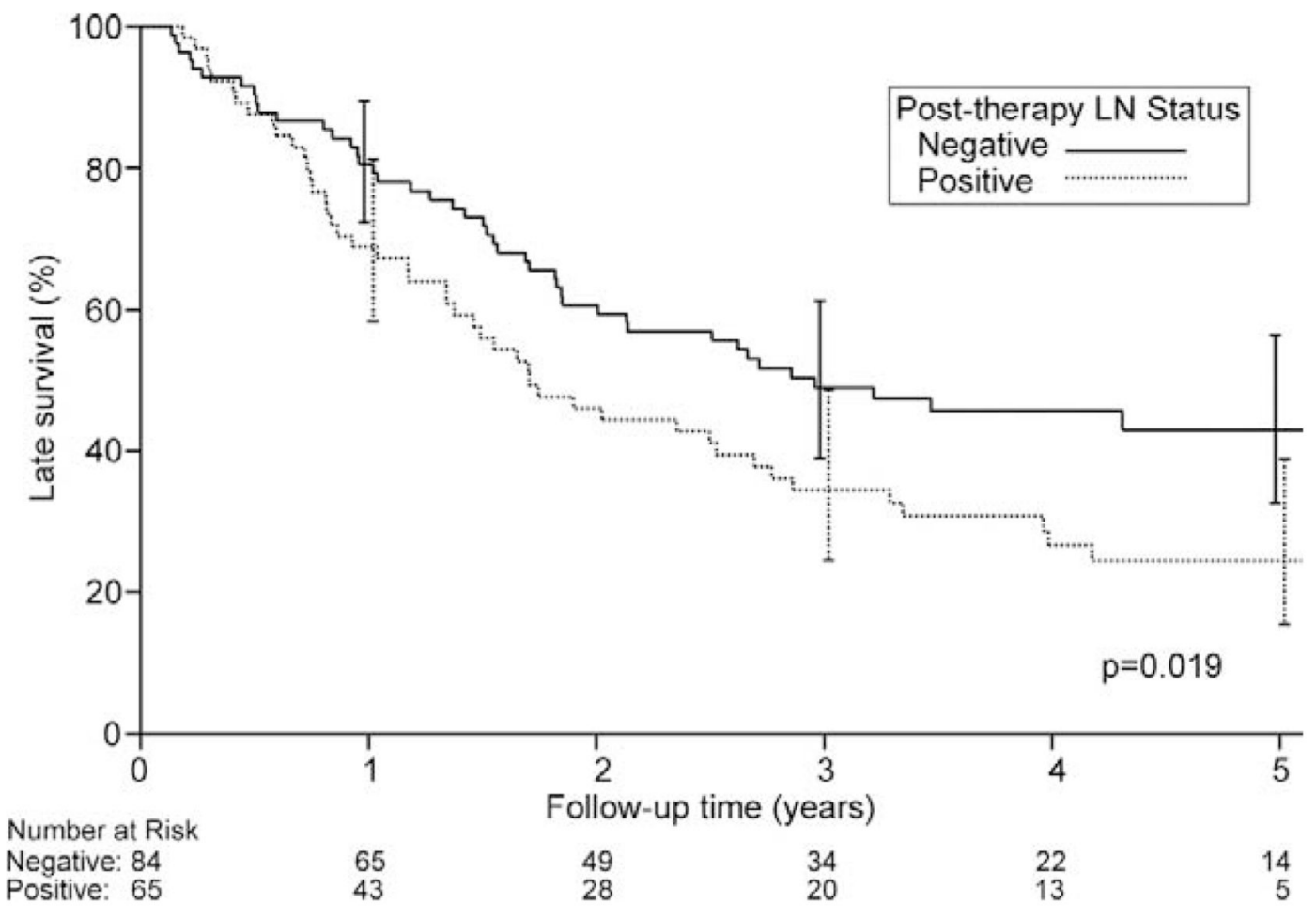

Fig 4.

Probability of overall survival (death from any cause) based on pathologic lymph node (LN) status in 149 operative survivors having neoadjuvant chemoradiotherapy followed by surgical resection. Zero time represents hospital discharge date. 


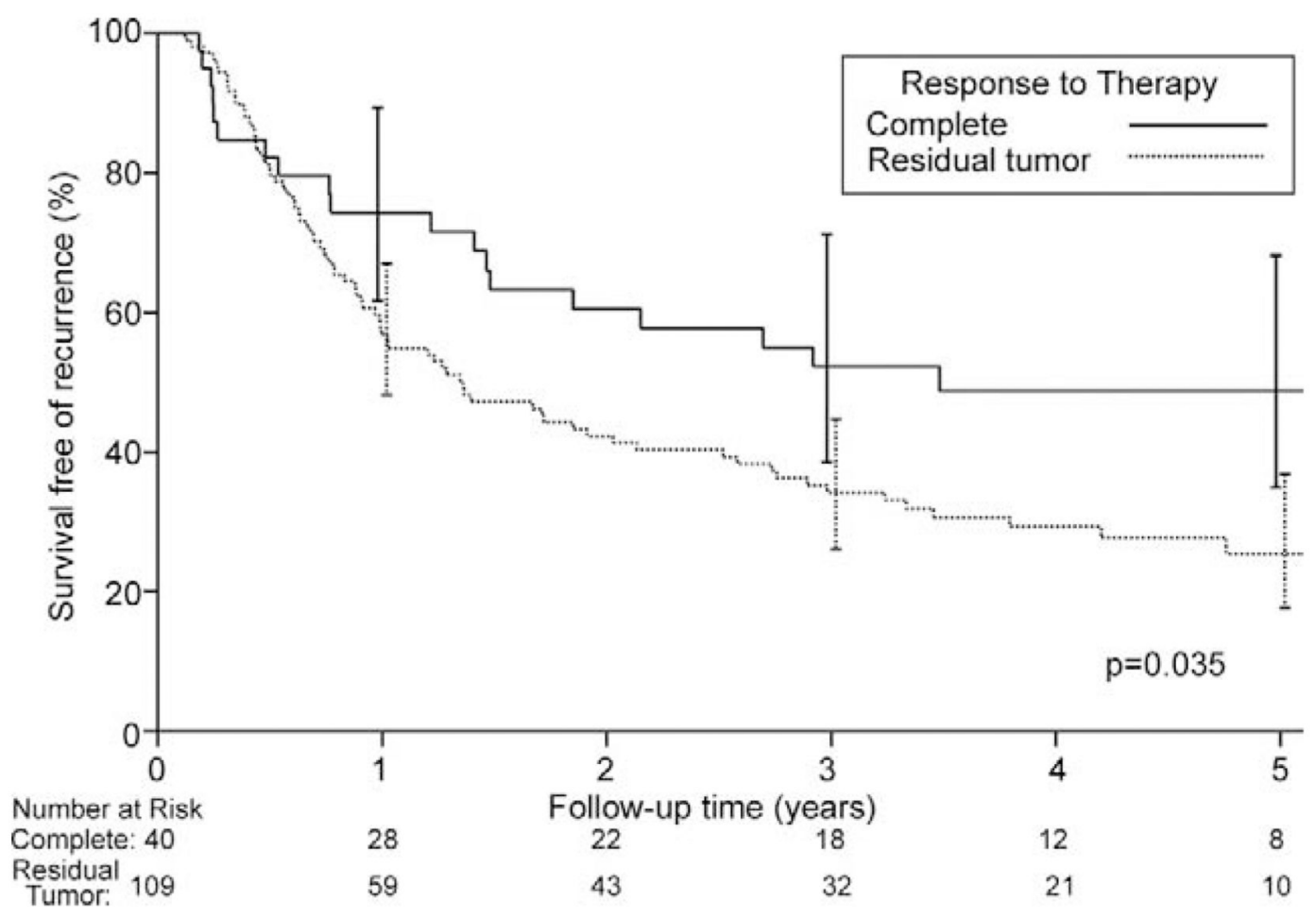

Fig 5.

Probability of disease-free survival (death from any cause) based on response to therapy in 149 operative survivors who underwent neoadjuvant chemoradiotherapy followed by surgical resection. Forty patients had a complete pathologic response, 109 patients had some degree of residual tumor present (either near complete or partial pathologic response). Zero time represents hospital discharge date. 


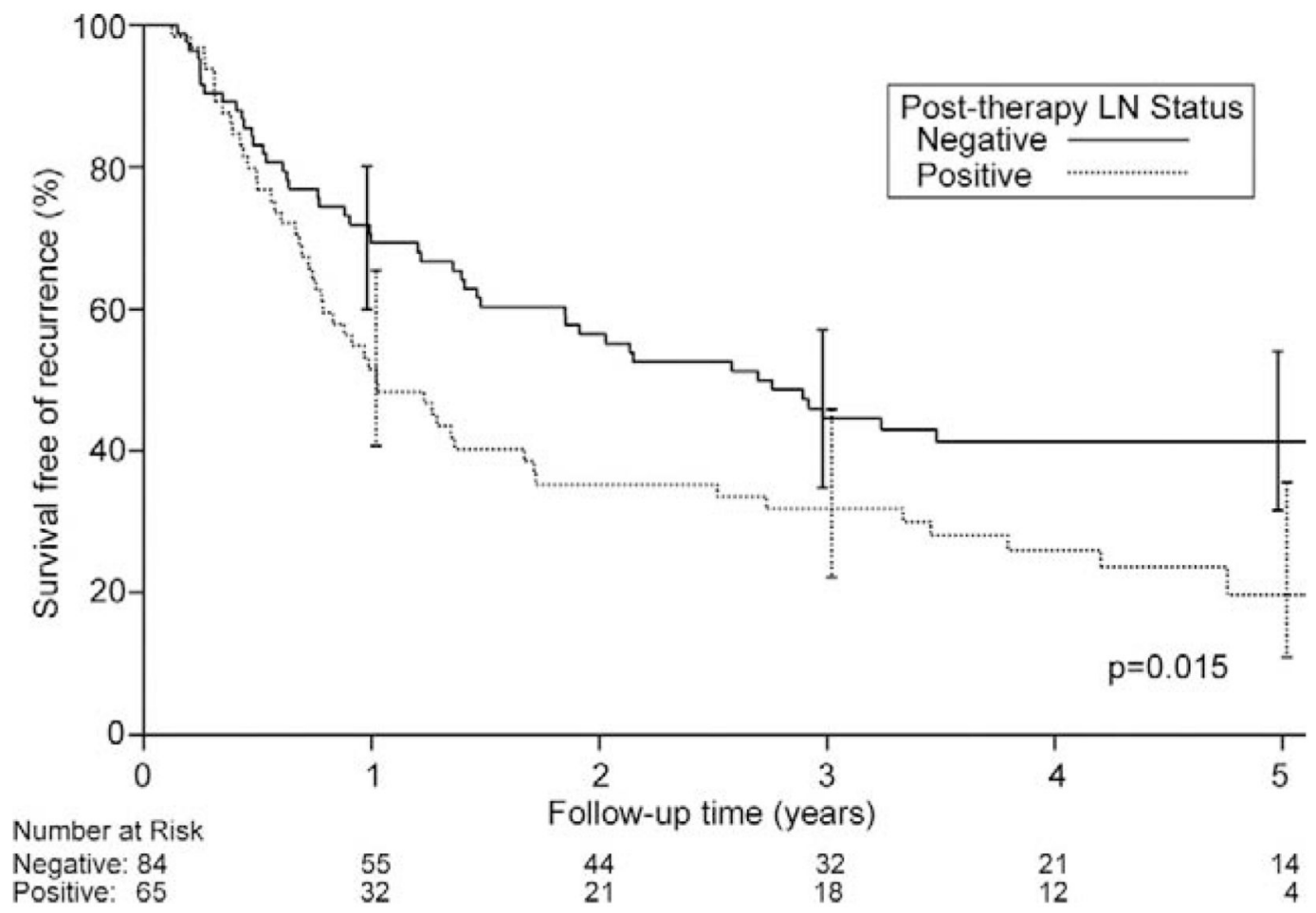

Fig 6.

Probability of disease-free survival (death from any cause) based on pathologic lymph node (LN) status in 149 operative survivors who underwent neoadjuvant chemoradiotherapy followed by surgical resection. Zero time represents hospital discharge date. 


\section{Table 1}

Prechemoradiation Therapy Clinical Stage

\begin{tabular}{llc}
\hline TNM Classification & Stage & Patients $(\%)$ \\
\hline T3N0M0 & IIA & $12(7.4)$ \\
T1N1M0 & IIB & $1(0.6)$ \\
T2N1M0 & IIB & $15(9.4)$ \\
T3N1M0 & III & $105(64.8)$ \\
T4N1M0 & III & $6(3.7)$ \\
T1N1M1a & IVA & $1(0.6)$ \\
T2N1M1a & IVA & $2(1.2)$ \\
T3N1M1a & IVA & $19(11.7)$ \\
T4N1M1a & IVA & $1(0.6)$ \\
\hline
\end{tabular}




\section{Table 2}

Postresection Pathologic Stage

\begin{tabular}{llc}
\hline TNM Classification & Stage & Patients (\%) \\
\hline T0N0M0 & 0 & $42(25.9)$ \\
T1N0M0 & I & $10(6.2)$ \\
T2N0M0 & IIA & $17(10.5)$ \\
T3N0M0 & IIA & $16(9.9)$ \\
T0N1M0 & IIB & $11(6.8)$ \\
T1N1M0 & IIB & $3(1.9)$ \\
T2N1M0 & IIB & $19(11.7)$ \\
T3N1M0 & III & $30(18.5)$ \\
TxNxM1 & IV & $5(3.1)$ \\
T0N0M1 & IV & $2(1.2)$ \\
T2N0M1 & IV & $1(0.6)$ \\
T2N1M1 & IV & $2(1.2)$ \\
T3N0M1 & IV & $1(0.6)$ \\
T3N1M1 & IV & $3(1.9)$ \\
\hline
\end{tabular}

Ann Thorac Surg. Author manuscript; available in PMC 2010 August 31. 


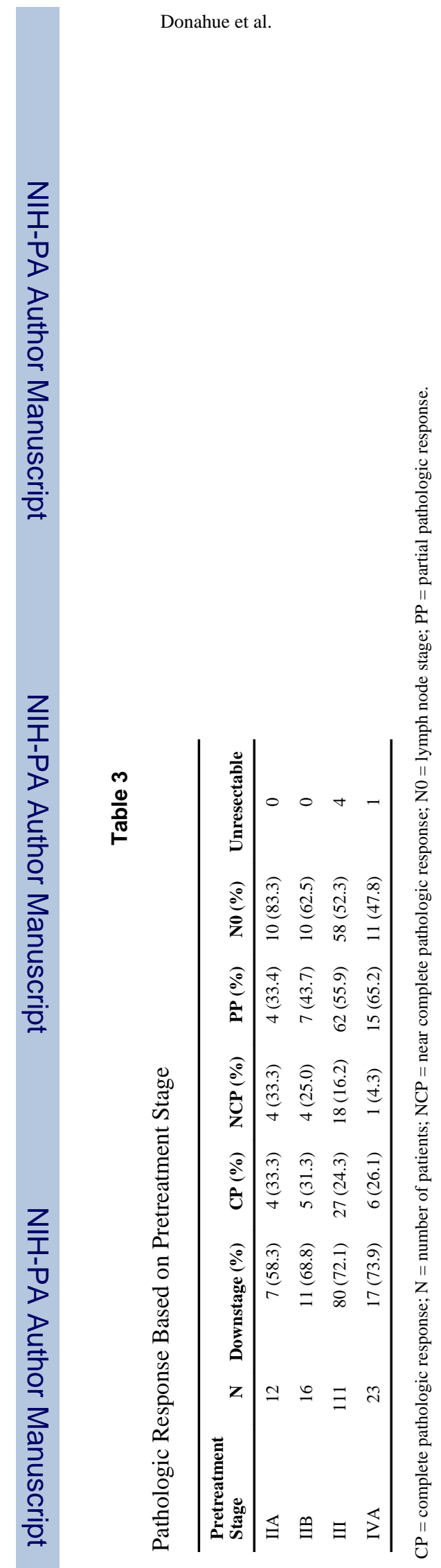

Ann Thorac Surg. Author manuscript; available in PMC 2010 August 31. 